S.Bitimkhan

Ye.A. Buketov Karaganda State University, Kazakhstan

(E-mail: bsamat10@mail.ru)

\title{
Hardy-Littlewood theorem for series with general monotone coefficients
}

In this work we study trigonometric series with general monotone coefficients. Also, we consider $L_{q} \varphi\left(L_{q}\right)$ space. In particular, when $\varphi(t) \equiv 1$ the space $L_{q} \varphi\left(L_{q}\right)$ coincides with $L_{q}$. Well known the theorem of Hardy and Littlewood about trigonometric series with monotone coefficients. Also known various generalizations of this theorem. In 1982 this theorem was generalized by M.F. Timan for the spaces $L_{q} \varphi\left(L_{q}\right)$. And in 2007 S.Tikhonov proved Hardy-Littlewood theorem for trigonometric series with general monotone coefficients. In this work we have generalized Hardy-Littlewood theorem for Fourier series of functions $f \in L_{q} \varphi\left(L_{q}\right)$ with general monotone coefficients. Also, obtained upper-bound estimate of best approximation of functions $f \in L_{q}$ through its Fourier's coefficients which are general monotone.

Keywords: trigonometric series, Hardy-Littlewood theorem, general monotone sequences, convergence, Fourier's coefficients.

Let $L_{q}(0,2 \pi), 1 \leq q<+\infty$ denotes the space of all $2 \pi$-periodic, measurable by Lebesgue functions $f(x)$, for which

$$
\|f\|_{q}=\left(\int_{0}^{2 \pi}|f(x)|^{q} d x\right)^{\frac{1}{q}}<+\infty .
$$

Through $E_{n}(f)_{q}$ we will designate the best approximation of a function $f \in L_{q}$ by trigonometrical polynomials of total degree $n$ in the metric of spaces $L_{q}$ :

$$
E_{n}(f)_{q}=\inf _{T_{n}}\left\|f-T_{n}\right\|_{q}
$$

Let the function $\varphi(t)$ satisfies the following conditions [1]:

a) $\varphi(t)$ is an even, non-negative, non-decreasing on $[0,+\infty)$;

b) $\varphi\left(t^{2}\right) \leq C \varphi(t), t \in[0, \infty), C \geq 1$;

c) $\frac{\varphi(t)}{t^{\varepsilon}} \downarrow$ on $(0,+\infty)$ for some $\varepsilon>0$.

Measurable, $2 \pi$-periodic function $f \in L_{q} \varphi\left(L_{q}\right)$, if

$$
\int_{0}^{\pi}|f(x)|^{q} \varphi\left(|f(x)|^{q}\right) d x<+\infty .
$$

In particular, when $\varphi(t) \equiv 1$ the space $L_{q} \varphi\left(L_{q}\right)$ coincides with $L_{q}$.

We consider the series

$$
\sum_{n=1}^{\infty} a_{n} \cos n x
$$

and denote by $f(x)$ the sum of this series.

Definition [2]. The sequence of numbers $\left\{a_{n}\right\}$ is said to be general monotone, or $\left\{a_{n}\right\} \in G M S$, if the relation

$$
\sum_{k=n}^{2 n-1}\left|a_{k}-a_{k+1}\right| \leq C\left|a_{n}\right|
$$

holds for all $n \geq 1$, where the constant $C$ is independent of $n$.

The set of all numerical sequences $\left\{a_{n}\right\}$ such that $a_{n} \downarrow 0, n \rightarrow \infty$, is denoted by $M S$. It is known that $M S \subset G M S$.

We give the following well-known theorem of Hardy-Littlewood 
Theorem $A[2]$. Let $\left\{a_{n}\right\} \in M S$. A necessary and sufficient condition that the function $f(x) \in L_{q}$, $1<q<+\infty$, is that

$$
\sum_{n=1}^{+\infty} n^{q-2} \cdot a_{n}^{q}<+\infty
$$

In 1982 this theorem was generalized by M.F.Timan [1] for the spaces $L_{q} \varphi\left(L_{q}\right)$.

Theorem B. Let $\varphi(t)$ satisfies conditions a)-c) and $f(x) \in L_{1}$ is an even function with Fourier series $\sum_{n=1}^{+\infty} a_{n} \cos n x$, where $\left\{a_{n}\right\} \in M S$. A necessary and sufficient condition that the function $f \in L_{p} \varphi\left(L_{p}\right)$ for some $p>1$, is that

$$
\sum_{n=1}^{+\infty} n^{p-2} \cdot a_{n}^{p} \varphi(n)<+\infty
$$

In 2007 S.Tikhonov [2] proved the following theorem.

Theorem $C$. Let $\left\{a_{n}\right\}$ be a positive sequence and $\left\{a_{n}\right\} \in G M S$. A necessary and sufficient condition that the function f should belong to $L_{q}, 1<q<+\infty$, is that inequality

$$
\sum_{n=1}^{+\infty} n^{q-2} \cdot a_{n}^{q}<+\infty
$$

holds.

Our main goal is to prove the theorem of Hardy and Littlewood for the Fourier series of a function $f \in L_{q} \varphi\left(L_{q}\right)$, the coefficients are generally monotonous.

To obtain the main result we need the following Lemma.

Lemma. Let $f(x)=\sum_{n=1}^{+\infty} a_{n} \cos n x$, where positive sequence $\left\{a_{n}\right\} \in G M S$ and for some $q, \quad 1<q<+\infty$ converges the series $\sum_{n=1}^{+\infty} n^{q-2} \cdot a_{n}^{q}$.

Then, the following inequality holds

$$
E_{n}(f)_{q} \leq C\left[a_{n}(n+1)^{1-\frac{1}{q}}+\left(\sum_{k=n+1}^{+\infty} k^{q-2} \cdot a_{k}^{q}\right)^{\frac{1}{q}}\right], n=1,2, \ldots
$$

Proof. From the properties of the best approximation and norms, we have

$$
\begin{gathered}
E_{n}(f)_{q} \leq\left\|f-S_{n}(f)\right\|_{q}=\left\|f-S_{n}(f)+a_{n} \cdot D_{n}(\cdot)-a_{n} \cdot D_{n}(\cdot)\right\|_{q} \leq \\
\leq\left\|f-S_{n}(f)+a_{n} \cdot D_{n}(\cdot)\right\|_{q}+a_{n}\left\|D_{n}(\cdot)\right\|_{q},
\end{gathered}
$$

where $S_{n}(f)$ is the partial sum of series $(1), D_{n}(x)=\sum_{k=1}^{n} \cos k x$ is the Dirichlet kernel.

For the Dirichlet kernel is known the following inequality [2]

$$
\left\|D_{n}(\cdot)\right\|_{q} \leq C \cdot n^{1-\frac{1}{q}} .
$$

To estimate the first term in (2) we use the theorem C. Because the sequence of coefficients of the series

$$
f(x)-S_{n}(f)(x)+a_{n} \cdot D_{n}(x)=\sum_{k=1}^{+\infty} b_{k} \cdot \cos k x
$$

belongs to GMS. Indeed, for

$$
b_{k}= \begin{cases}a_{n}, & k=1, \ldots, n \\ a_{k}, & k=n+1, \ldots\end{cases}
$$

we have

$$
\sum_{k=m}^{2 m-1}\left|b_{k}-b_{k+1}\right|=0<C \cdot b_{m}, \text { at } 2 m-1 \leq n
$$




$$
\sum_{k=m}^{2 m-1}\left|b_{k}-b_{k+1}\right|=\sum_{k=m}^{2 m-1}\left|a_{k}-a_{k+1}\right| \leq C \cdot a_{m}=C \cdot b_{m}, \text { at } m \geq n .
$$

If $m<n<2 m-1$, then we assume that $b_{k}=a_{n}, k=m, \ldots, m+s$ and $b_{k}=a_{k}, k=m+s+1, \ldots, 2 m-1$, where $s$ is natural number. Then

$$
\begin{aligned}
& \sum_{k=m}^{2 m-1}\left|b_{k}-b_{k+1}\right|=\sum_{k=m}^{m+s-1}\left|b_{k}-b_{k+1}\right|+\sum_{k=m+s}^{2 m-1}\left|b_{k}-b_{k+1}\right|= \\
= & \sum_{k=m+s}^{2 m-1}\left|a_{k}-a_{k+1}\right|<\sum_{k=m+s}^{2(m+s)-1}\left|a_{k}-a_{k+1}\right| \leq C \cdot a_{m+s} \leq C \cdot b_{m} .
\end{aligned}
$$

Therefore, by theorem $\mathrm{C}$

$$
\begin{aligned}
\| f-S_{n}(f) & +a_{n} \cdot D_{n}(\cdot) \|_{q} \leq C\left(a_{n}^{q} \sum_{k=1}^{n} k^{q-2}+\sum_{k=n+1}^{\infty} a_{k}^{q} \cdot k^{q-2}\right)^{\frac{1}{q}} \leq \\
& \leq C \cdot a_{n}(n+1)^{1-\frac{1}{q}}+\left(\sum_{k=n+1}^{\infty} a_{k}^{q} \cdot k^{q-2}\right)^{\frac{1}{q}} .
\end{aligned}
$$

Now, using inequalities (3) and (4), from (2) we have

$$
E_{n}(f)_{q} \leq C\left[a_{n}(n+1)^{1-\frac{1}{q}}+\left(\sum_{k=n+1}^{+\infty} k^{q-2} \cdot a_{k}^{q}\right)^{\frac{1}{q}}\right], n=1,2, \ldots
$$

This completes the proof of Lemma.

Now we prove the main result:

Theorem. Let the function $\varphi(t)$ satisfies the conditions a)-c), and $f(x) \in L_{1}$ is an even function with Fourier series $\sum_{n=1}^{+\infty} a_{n} \cos n x$, where $\left\{a_{n}\right\}$ is positive sequence, and $\left\{a_{n}\right\} \in G M S$.

A necessary and sufficient condition that the function $f$ should belong to $L_{q} \varphi\left(L_{q}\right), q>1$, is that

$$
\sum_{n=2}^{+\infty} n^{q-2} \cdot a_{n}^{q} \cdot \varphi(n)<+\infty
$$

Proof. Suppose inequality (5) holds. Then, from the properties of the function $\varphi(t)$ converges also following series

$$
\sum_{n=2}^{+\infty} n^{q-2} \cdot a_{n}^{q}
$$

So, by theorem 4.2 of [2] $f \in L_{q}$. Then applying the Lemma, the inequality of Hardy [1] and the properties of the function $\varphi(t)$, we have for $1<p_{0}<q<+\infty$ :

$$
\begin{gathered}
\sum_{n=1}^{+\infty} n^{\frac{q}{p_{0}}-2} \cdot \varphi(n) \cdot E_{n}^{q}(f)_{p_{0}} \leq C \cdot \sum_{n=1}^{+\infty} n^{\frac{q}{p_{0}}-2} \cdot \varphi(n) \cdot\left[a_{n}(n+1)^{1-\frac{1}{p_{0}}}+\left(\sum_{k=n+1}^{+\infty} k^{p_{0}-2} \cdot a_{k}^{p_{0}}\right)^{\frac{1}{p_{0}}}\right]^{q} \leq \\
\leq C \cdot \sum_{n=1}^{+\infty} n^{\frac{q}{p_{0}}-2} \cdot \varphi(n) \cdot a_{n}^{q} n^{q\left(1-\frac{1}{p_{0}}\right)}+C \cdot \sum_{n=1}^{+\infty} n^{\frac{q}{p_{0}}-2} \cdot \varphi(n)\left(\sum_{k=n+1}^{+\infty} k^{p_{0}-2} \cdot a_{k}^{p_{0}}\right)^{\frac{q}{p_{0}}} \leq \\
\leq C \cdot \sum_{n=1}^{+\infty} n^{q-2} \cdot \varphi(n) \cdot a_{n}^{q}+C \cdot \sum_{n=1}^{+\infty} n^{-\left(2-\frac{q}{p_{0}}\right)}\left(\sum_{k=n+1}^{+\infty} k^{p_{0}-2} \cdot a_{k}^{p_{0}} \varphi^{\frac{p_{0}}{q}}(k)\right)^{\frac{q}{p_{0}}} \leq
\end{gathered}
$$




$$
\begin{gathered}
\leq C \cdot \sum_{n=1}^{+\infty} n^{q-2} \cdot \varphi(n) \cdot a_{n}^{q}+C \cdot \sum_{n=1}^{+\infty} n^{\frac{q}{p_{0}}-2}\left(n \cdot a_{n}^{p_{0}} \varphi^{\frac{p_{0}}{q}}(n) \cdot n^{p_{0}-2}\right)^{\frac{q}{p_{0}}} \leq \\
\leq C \cdot \sum_{n=1}^{+\infty} n^{q-2} \cdot a_{n}^{q} \varphi(n)<+\infty .
\end{gathered}
$$

Hence by theorem 3.3 of [1] we have

$$
\int_{0}^{\pi}|f(x)|^{q} \varphi\left(|f(x)|^{q}\right) d x<+\infty .
$$

Now let us prove the opposite. Let $f(x) \in L_{q} \varphi\left(L_{q}\right)$. Then by theorem 13.1 of [3; 54] we have

$$
\int_{0}^{\pi}|f(x)|^{q} \varphi\left(|f(x)|^{q}\right) d x=\int_{0}^{\pi}\left(f^{*}(t)\right)^{q} \varphi\left(\left(f^{*}(t)\right)^{q}\right) d t<+\infty,
$$

where $f^{*}$ - is non-increasing rearrangement of $f$.

Let

$$
f_{1}(x)=\int_{0}^{x} f(u) d u \text { and } f_{2}(x)=\int_{0}^{x} f_{1}(u) d u, \text { for } x \in(0, \pi) .
$$

Also for $x \in(0, \pi)$ we denote

$$
f_{3}(x)=\int_{0}^{x} f^{*}(u) d u \text { and } f_{4}(x)=\int_{0}^{x} f_{3}(u) d u .
$$

In [4] proved that for $\frac{\pi}{4(n+1)} \leq x \leq \frac{\pi}{4 n}$ the following inequality is satisfied

$$
f_{4}(x) \geq\left|f_{2}(x)\right| \geq f_{2}(x) \geq \frac{C}{n^{2}} \sum_{k=\left[\frac{n}{2}\right]}^{n} a_{k} .
$$

Next, arguing as in [4], we have

$$
\begin{gathered}
\sum_{n=1}^{+\infty} n^{q-2} \cdot a_{n}^{q} \varphi(n) \leq C \cdot \sum_{n=1}^{+\infty} n^{q-2} \cdot \varphi(n)\left(\frac{2}{n} \sum_{k=\left[\frac{n}{2}\right]}^{n} a_{k}\right)^{q}= \\
=C \cdot \sum_{n=1}^{+\infty} n^{-2} \cdot \varphi(n)\left(\sum_{k=\left[\frac{n}{2}\right]}^{n} a_{k}\right)^{q} \leq C \cdot \sum_{n=1}^{+\infty} n^{2 q-2} \cdot \varphi(n)_{\frac{\pi}{4(n+1)} \leq x \leq \frac{\pi}{4 n}}\left(f_{4}(x)\right)^{q} \leq \\
\leq C \cdot \sum_{n=1}^{+\infty} \varphi(n) \frac{4 n(n+1)}{\pi} \int_{\frac{\pi}{4(n+1)}}^{\frac{\pi}{4 n}}\left(\frac{\pi}{4 x}\right)^{2 q-2}\left(f_{4}(x)\right)^{q} d x \leq \\
\leq C \cdot \sum_{n=1}^{+\infty} \int_{\frac{\pi}{4(n+1)}}^{\frac{\pi}{4 n}}\left(\frac{\pi}{4 x}\right)^{2 q} \varphi\left(\frac{\pi}{x}\right)\left(f_{4}(x)\right)^{q} d x \leq \\
\leq C \cdot \int_{0}^{\pi} x^{-2 q} \varphi\left(\frac{\pi}{x}\right)\left(\int_{0}^{x} f_{3}(u) d u\right)^{q} d x= \\
=C \int_{0}^{\pi} x^{-2 q} \varphi\left(\frac{\pi}{x}\right)\left(\int_{0}^{x}\left(\int_{0}^{u} f^{*}(t) d t\right) d u\right)^{q} d x \leq \\
\leq C \int_{0}^{\pi} x^{-q}\left(\int_{0}^{x}\left(\int_{0}^{u} \varphi^{\frac{1}{q}}\left(\frac{\pi}{t}\right) f^{*}(t) d t\right) \frac{d u}{u}\right)^{q} d x \leq \\
\leq C \int_{0}^{\pi} x^{-q+1}\left(\int_{0}^{x}\left(\int_{0}^{u} \varphi^{\frac{1}{q}}\left(\frac{\pi}{t}\right) f^{*}(t) d t\right) \frac{d u}{u}\right)^{q} \frac{d x}{x}=
\end{gathered}
$$




$$
\begin{gathered}
=C \int_{0}^{\pi}\left(x^{-1+\frac{1}{q}} \int_{0}^{x}\left(\int_{0}^{u} \varphi^{\frac{1}{q}}\left(\frac{\pi}{t}\right) f^{*}(t) d t\right) \frac{d u}{u}\right)^{q} \frac{d x}{x} \leq \\
\leq C \int_{0}^{\pi}\left(x^{-1+\frac{1}{q}} \int_{0}^{x} \varphi^{\frac{1}{q}}\left(\frac{\pi}{t}\right) f^{*}(t) d t\right)^{q} \frac{d x}{x}= \\
=C \int_{0}^{\pi}\left(\frac{1}{x} \int_{0}^{x} \varphi^{\frac{1}{q}}\left(\frac{\pi}{t}\right) f^{*}(t) d t\right)^{q} d x \leq C \cdot \int_{0}^{\pi} \varphi\left(\frac{\pi}{t}\right) \cdot\left(f^{*}(t)\right)^{q} d t \leq \\
\leq C \cdot \int_{0}^{\pi}\left(f^{*}(t)\right)^{q} \varphi\left(\left(f^{*}(t)\right)^{q}\right) d t<+\infty .
\end{gathered}
$$

This completes the proof of Theorem.

Remark. The proved Theorem is extension of the Theorem B. Also, at $\varphi(t) \equiv 1$ the Theorem C follows from the proved Theorem.

\title{
References
}

1 Тиман М.Ф. Некоторые дополнения к теоремам вложения П.Л. Ульянова / М.Ф. Тиман // Депонир. ВИНИТИ. - 1982. - № 1750-82. - 17 с.

2 Tikhonov S. Trigonometric series with general monotone coefficients / S.Tikhonov // Journal Math. Anal. Appl. - 2007. - 326. - P. 721-735.

3 Зигмунд А. Тригонометрические ряды / А.Зигмунд. - М.: Мир, 1965. - Т. 1. - 615 с.

4 Booton B. General monotone sequences and trigonometric series / B.Booton // Math. Nachr. - 2014. Vol. 287. - No. 5, 6. - P. 518-529.

\section{С.Бітімхан}

\section{Жалпы монотонды коэффициентті қатарлар үшін Харди-Литтлвуд теоремасы}

\begin{abstract}
Мақалада жалпы монотонды коэффициентті тригонометриялық қатарлар зерттелді. Сонымен бірге $L_{q} \varphi\left(L_{q}\right)$ кеңістігі қарастырылды. Дербес жағдайда $\varphi(t) \equiv 1$ болғанда $L_{q} \varphi\left(L_{q}\right)$ кеңістігі $L_{q}$ кеңістігімен беттеседі. Монотонды коэффициентті тригонометриялық қатарлар үшін Харди мен Литтлвуд теоремасы жақсы белгілі. Сондай-ақ теореманың әртүрлі жалпыламалары да белгілі. 1982 ж. осы теореманы М.Ф.Тиман $L_{q} \varphi\left(L_{q}\right)$ кеңістігі үшін жалпылады, ал 2007 ж. С.Тихонов Харди-Литтлвуд теоремасын жалпы монотонды коэффициентті тригонометриялық қатарлар үшін дәлелдеді. Бұл жұмыста Харди-Литтлвуд теоремасын $f \in L_{q} \varphi\left(L_{q}\right)$ функциясының коэффициенттері жалпы монотонды болатын Фурье қатарлары үшін жалпыланды. Сонымен бірге $f \in L_{q}$ функциясының ең жақсы жуықтауының жоғарыдан бағалауын оның жалпы монотонды болатын Фурье коэффициенттері арқылы алынды.
\end{abstract}

Kiлm сөздер: тригонометриялық қатарлар, Харди-Литтлвуд теоремасы, жалпы монотонды тізбектер, жинақтылық, Фурье коэффициенттері. 


\section{С.Битимхан}

\section{Теорема Харди-Литтлвуда для рядов с обобщенно- монотонными коэффициентами}

В статье исследованы тригонометрические ряды с обобщенно-монотонными коэффициентами. Также рассмотрено пространство $L_{q} \varphi\left(L_{q}\right)$. В частности, когда $\varphi(t) \equiv 1$, пространство $L_{q} \varphi\left(L_{q}\right)$ совпадает с $L_{q}$. Хорошо известна теорема Харди и Литтлвуда о тригонометрических рядах с монотонными коэффициентами. Также известны различные обобщения этой теоремы. В 1982 г. М.Ф.Тиман обобщил эту теорему для пространства $L_{q} \varphi\left(L_{q}\right)$, а в 2007 г. С.Тихонов доказал теорему Харди-Литтлвуда для тригонометрических рядов с обобщенно-монотонными коэффициентами. В данной работе обобщили теорему Харди-Литтлвуда для рядов Фурье функции $f \in L_{q} \varphi\left(L_{q}\right)$ с обобщенно-монотонными коэффициентами. Также получена верхняя оценка наилучшего приближения функции $f \in L_{q}$ через её коэффициенты Фурье, которые являются обобщенно-монотонными.

Ключевые слова: тригонометрические ряды, теорема Харди-Литтлвуда, обобщенно-монотонные последовательности, сходимость, коэффициенты Фурье.

\section{References}

1 Timan, M.F. (1982). Nekotorye dopolnenia k teoremam vlozhenia P.L.Ulianova [Some additions to embedding theorems of P.L.Uljyanov]. Deponirovanie VINITI, 1750-82, 17 [in Russian].

2 Tikhonov, S. (2007). Trigonometric series with general monotone coefficients. J. Math. Anal. Appl., 326, $721-735$.

3 Zygmund, A. (1965). Trihonometricheskie riady [Trigonometric series]. (Vol. I, 615). Moscow: Mir [in Russian].

4 Booton, B. (2014). General monotone sequences and trigonometric series. Math. Nachr., 287, No. 5, 6, $518-529$. 